\title{
Subaru Hyper Suprime Cam Survey of the Andromeda Halo
}

\author{
Masashi Chiba ${ }^{1}$, Mikito Tanaka ${ }^{1}$ and Yutaka Komiyama ${ }^{2}$ \\ ${ }^{1}$ Astronomical Institute, Tohoku University, Sendai, Japan \\ email: chiba@astr.tohoku.ac.jp \\ ${ }^{2}$ NAOJ, Mitaka, Tokyo, Japan
}

\begin{abstract}
We present a progress report on our deep and wide-field imaging survey of the Andromeda halo with Hyper Suprime Cam (HSC) mounted on Subaru. HSC is the upgraded prime focus camera after Suprime-Cam, having a field of view of 1.77 square degree (1.5 degree in diameter), namely about 10 times larger than that of Suprime-Cam. This camera will thus offer us great opportunities to explore unique and legacy surveys for the Andromeda halo, as well as for other Galactic Archaeology science cases.
\end{abstract}

Keywords. Galactic Archaeology, Subaru/HSC, M31

\section{Deep and wide-field survey of the M31 halo}

We are now carrying out intensive observing programs for a deep and wide-field imaging survey of M31's stellar halo using an HSC imager. This extremely wide-field camera in combination with Subaru will reach a depth of about 1.5 mag fainter than the Horizontal Branch (HB) magnitude ( $\sim 27.4$ and $\sim 26.4$ mag in $g$ and $i$ bands, respectively) to obtain numerous HB stars, which will allow us to identify and characterize low surface brightness features as faint as $\mu$ of $33.5 \mathrm{mag} \operatorname{arcsec}^{-2}$. This surface brightness is significantly lower than was possible with previous surveys that were based entirely on the selection of bright red giant branch (RGB) stars. It is thus possible to find and map out faint stellar streams even in the outer part of the halo at $R$ beyond $90 \mathrm{kpc}$, where the very low density of bright RGB stars makes finding streams very difficult. Similarly, this Subaru/HSC survey will allow us to find lower luminosity dwarf satellites than hitherto possible through member HB stars, thereby providing important insight into the missing satellite problem.

This extremely wide-field camera will cover about $80 \mathrm{kpc}$ x $80 \mathrm{kpc}$ area (6 deg x $6 \mathrm{deg}$ ) in the north-west part of the M31 halo (Fig. 1), which is wide enough to contain a large portion of the outer halo. As back-up observations (for the nights with non-ideal seeing conditions), short-exposure imaging surveys for the rest of the halo areas in M31 are made to obtain the data of bright RGB stars in combination of the use of the narrow-band filter, NB515. The stars selected in this mode will be targets for our future spectroscopic follow-up using Prime Focus Spectrograph to be mounted on Subaru (see Chiba et al. 2015 in this volume).

\section{Current status}

In period S14B, we succeeded to obtain $g$ and $i$ deep imaging only for two HSC fields and yet slightly incomplete exposures for one field with $\sim 0.5$ mag deficient compared with other two fields (filled circles in Fig. 1), due to the bad weather. The left panel of Fig. 2 shows the CMD of the stars in these three fields. It is clear that Subaru/HSC is quite powerful for imaging M31 stars as faint as $g=27.4$ and $i=26.4$, and successful 


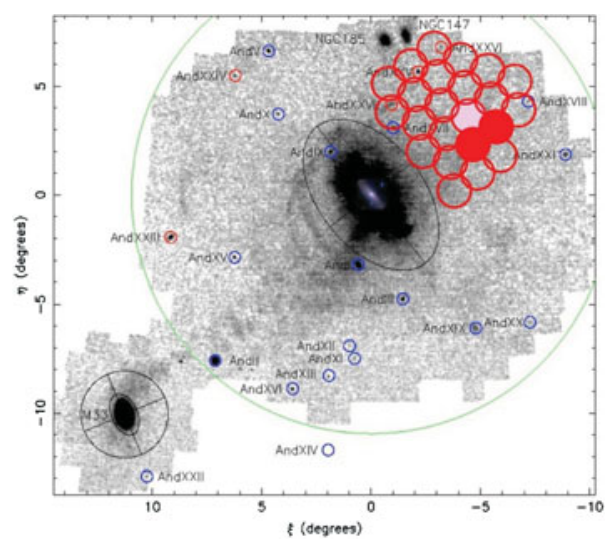

Figure 1. Proposed survey fields with HSC in M31's halo overlaid onto the RGB map of PAndAS. Filled circles denote the fields for which $g$ - and $i$-band deep imagings were completed and partially completed, respectively, in S14B.
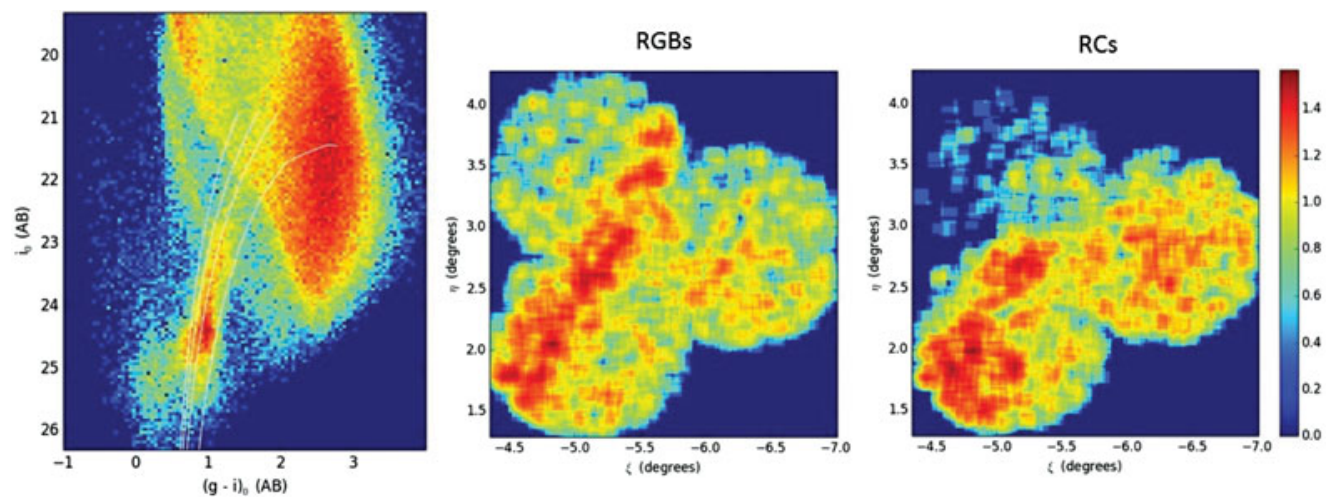

Figure 2. Left: $i$ vs. $(g-i)$ color-magnitude diagram obtained from three HSC fields (filled circles in Fig. 1) in S14B. Middle: RGB map in these three fields selected with $22.7<i<24.0$. Right: RC map selected with $24.1<i<24.7$. Note that in the RC map, the northern stream in the upper-left HSC pointing disappears due to yet inefficient exposures and S/N.

for hunting Red Clump (RC) stars with $i \sim 24.5$ in HB; clearly the previous survey data with $i<24$ using 4m-class telescopes are too shallow and largely affected by the contamination of the MW foreground stars (as seen in the upper right region, peaked at $i<24$ and $2<g-i<3$ ), thus showing only the brightest part of the M31 halo with some uncertainties associated with this contamination issue. The middle and right panels in Fig. 2 show the spatial distributions of RGB and RC stars, respectively. It is remarkable that while both of these maps show stream-like features (from lower left to upper middle) corresponding to the part of the northern stream, the RC map shows additional faint substructures, which do not appear in the RGB map. Further HSC observations of M31's halo are scheduled to assess and limit these overdensities. We have also identified some systematic difference in RC magnitudes between the stars in and outside the northern stream, which reflects the different metallicities and ages. Deep imaging indeed enables us to discover new important features in M31's halo. 